\title{
The Missing Piece in Glaucoma?
}

\author{
Syed S. Hasnain \\ General Ophthalmology, Porterville, CA, USA \\ Email: hasnain40@sbcglobal.net
}

Received 20 January 2016; accepted 26 February 2016; published 29 February 2016

Copyright (C) 2016 by author and Scientific Research Publishing Inc.

This work is licensed under the Creative Commons Attribution International License (CC BY).

http://creativecommons.org/licenses/by/4.0/

(c) ()

\section{Abstract}

Glaucoma is defined as an optic disc neuropathy meaning the nerve fibers are being atrophied similar to the fate occurring in non-glaucomatous optic atrophies. Furthermore, the nerve fibers are always being destroyed randomly in all the non-glaucomatous optic atrophies. In contrast, the nerve fibers in glaucoma are invariably destroyed in an orderly tandem fashion, from peripheral to central, never randomly. Is glaucoma really an optic disc neuropathy in light of orderly destruction of nerve fibers in glaucoma? The current prevailing theories in glaucoma such as posterior bowing of the lamina cribrosa or cupping can't explain the orderly destruction of nerve fibers occurring in glaucoma. In fact, there is no biological mechanism acting directly on the nerve fibers or their RGCs which could lead to their orderly destruction. Therefore, there should be some mechanical way, which could result in the orderly destruction of nerve fibers even though this mechanical scenario may have resulted from the direct biological effect of raised IOP on some important component of the optic disc. It is proposed that the border tissue of Elschnig (BT) atrophies due to chronic ischemia caused by raised IOP, and as a result, the lamina cribrosa (LC) begins sinking in the scleral canal-a mechanical problem. Due to sinking of the LC, the nerve fibers get stretched and broken starting with the most peripheral nerve fibers being closest to the edge of the scleral opening and ending with the most central nerve fibers in an orderly tandem fashion. Therefore, in view of the orderly destruction of nerve fibers, glaucoma may not be an optic disc neuropathy but an optic disc axotomy.

\section{Keywords}

Glaucoma, Normal Tension Glaucoma, Severance, Arcuate Field Defects, Disc Notching, Disc Hemorrhage, Sinking Disc, Cupping Disc, Excavated Disc, RNFL

\section{Introduction}

Chronic glaucoma has been a mystery ever since it was given a separate entity 160 years ago [1]. Ophthalmolo- 
gists of the time found the optic discs of chronic glaucoma subjects being cupped, instead of being normally flat. It is presumed that the disc becomes cupped due to the force of raised intraocular pressure (IOP) resulting in atrophy and shrinkage of the nerve fibers. Until now, cupping of the disc and atrophy of the nerve fibers are still considered the salient features of the glaucomatous disc and thus glaucoma is defined as an optic disc neuropathy.

However, the concept of cupping disc and atrophy of the nerve fibers has failed to answer many pathognomonic features of glaucoma including predictable visual field defects. I believe the concept of cupping was given mistakenly in the 1850s, which has put us on the wrong path in glaucoma. One hundred years later, the term cup/disc ratio was introduced which gave further credence to the cupping concept but at the same time created conundrum in glaucoma diagnosis since ironically we started using the same parameter of "cupping" in describing both physiological as well as glaucomatous cupping of the disc.

It is imperative to mention the arrangement of nerve fibers in this discussion. There are three main aspects in which the nerve fibers are arranged in the retina and in the optic disc. First, the nerve fibers in the retina are arranged in layers superficial to deep. Second, the most central vision fibers originate closest to the disc, like most superficial (closest to vitreous) and exit from the most central part of the disc. In contrast, the most peripheral nerve fibers originate from the most distant retina or farthest from the optic disc, lie deepest (closest to sclera) and exit closest to the edge of the scleral opening, Figure 1. Third, the nerve fibers originating from the nasal retina proceed directly to the nasal part of the optic disc. However, the situation is different in the temporal retina because of the presence of the macular fibers. The nerve fibers originating from the nasal aspect of the macular area proceed directly to the central temporal part of the disc. The fibers originating from the temporal macular and peripheral retina have to arch above and below the macular fibers to reach the superior and inferior poles of the optic disc respectively and hence are known as the arcuate fibers.

We may differ on many aspects in glaucoma but on one issue we all have consensus: that the million or so nerve fibers densely packed in a disc are always invariably being destroyed, one by one, from peripheral to central in an orderly tandem fashion, and never randomly. If the nerve fibers are not destroyed in a predictable orderly sequence, the role of visual field tests in glaucoma would be meaningless.

The cupping disc/atrophy of the nerve fibers paradigm has failed to explain the orderly destruction of nerve fibers [2], which is hallmark of glaucoma. For any glaucoma theory to prevail, it must explain the orderly destruction of nerve fibers otherwise it will not be valid. In light of the orderly destruction of nerve fibers all the prevailing glaucoma theories such as the direct role of raised IOP, apoptosis, neurodegeneration [3], increased sensitivity of the disc to IOP, posterior bowing of the lamina cribrosa or cupping become wrong as none of them can explain the orderly destruction of nerve fibers occurring in glaucoma. If the nerve fibers are being destroyed

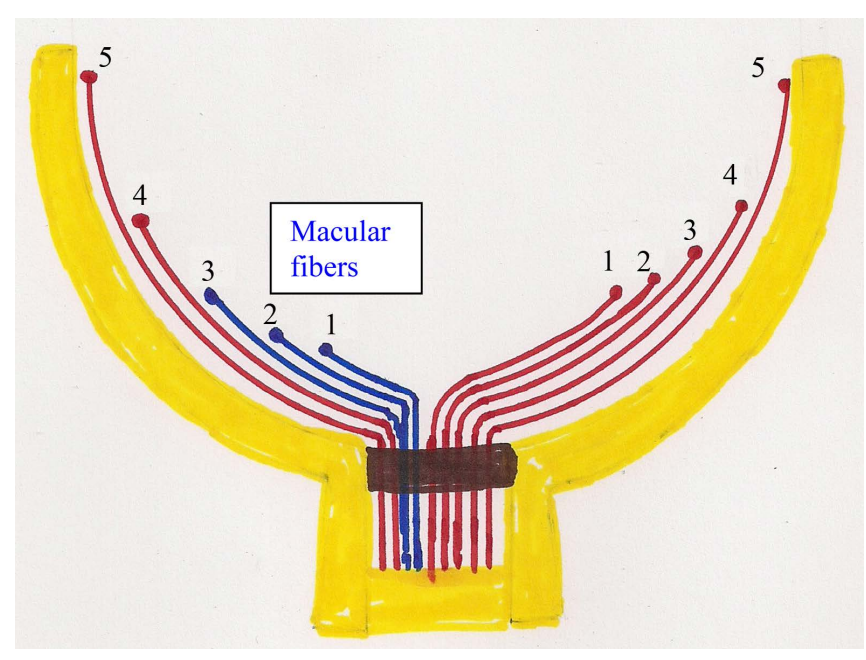

Figure 1. Arrangement of nerve fibers in the retina and optic disc. Most peripheral fiber (5) originates farthest from the disc, lies closest to the sclera and exit closest to the scleral edge. Most central fiber (1) originates closest to the disc, lies closest to vitreous and exits from the most central part of scleral opening. 
in an orderly tandem fashion in glaucoma, then we should expect the mechanism for their destruction to be orderly as well.

In fact, there is no biological mechanism which acting directly on the nerve fibers or their RGCs could result in their orderly destruction. Therefore, for the orderly destruction of nerve fibers to occur in glaucoma, there must be some mechanical way even though that mechanical scenario may have resulted from the direct biological effect of raised IOP on some very important component of the optic disc.

\section{What May Be the Mechanical Scenario?}

The border tissue of Elschnig (BT) keeps the LC firmly in place in the scleral opening. The LC is sinking due to atrophy of the border tissue which is solely supplied by ciliary circulation. Systemic circulatory pressure supplying the BT and IOP are opposing forces. Normally, the circulatory pressure supplying the BT should be higher than the IOP for the proper perfusion and healthy maintenance of the BT, Figure 2.

However, if this delicate situation is reversed, either due to raised IOP or if pressure supplying the BT becomes lower than the IOP due to systemic problems such as chronic hypotension, then even normal range IOP can take the upper hand. Thus, even the normal range IOP will begin compressing the circulation of the BT thereby inducing chronic ischemia and its atrophy and NTG will ensue. Therefore, it is the IOP becoming higher than the circulatory pressure of the BT resulting in both HTG and NTG.

The eyeball is supplied by dual circulation, the central retinal artery (CRA) — a high pressure system and ciliary circulation - a comparatively lower pressure system. In acute glaucoma, when IOP exceeds pressure of the CRA, it compresses circulation of the retina resulting in immediate death of neuronal tissue and optic atrophy. Furthermore, the optic atrophy resulting from acute glaucoma is a flat disc (non-excavated) as there is no sinking of the LC and thus no severance of nerve fibers taking place.

In contrast, chronic glaucoma develops when IOP becomes higher than the ciliary pressure supplying the BT. Since the ciliary pressure is a lower pressure system, even a moderate elevation of IOP can become higher than ciliary pressure and chronically compress circulation of the BT, resulting in its atrophy. As the BT beocmes atrophied, the LC begins sinking and the nerve fibers get stretched and severed at the scleral edge. In summary,

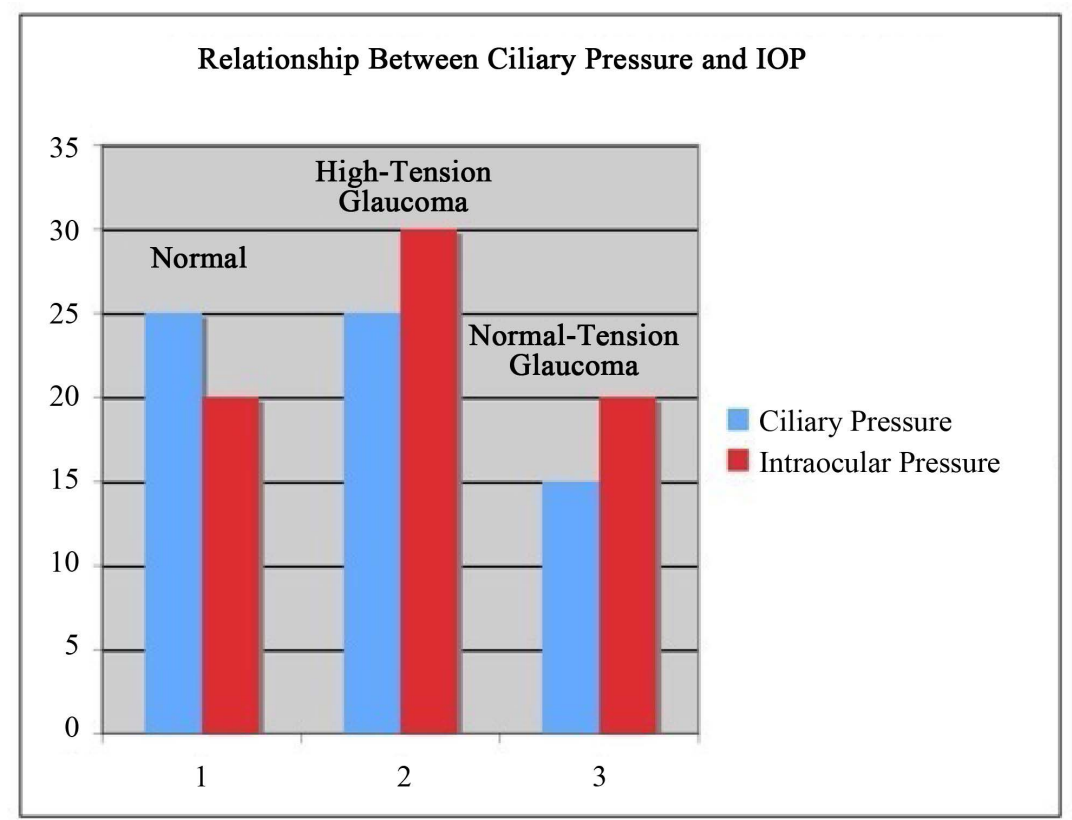

Figure 2. Relationship between ciliary pressure and IOP. Normally, ciliary circulatory pressure supplying the border tissue should be higher than IOP for healthy perfusion as in column (1). In column (2), the IOP is increased to 30 whereas the ciliary pressure remains the same at 25 , this will result in high-tension glaucoma. In column (3) the ciliary pressure is decreased to $15 \mathrm{~mm}$ but the IOP is same, normal at 20 , resulting in normal-tension glaucoma. 
the nerve fibers are being atrophied in a non-orderly fashion in acute glaucoma whereas, the nerve fibers are being severed in an orderly fashion in chronic glaucoma.

Due to atrophy of the BT, the LC starts sinking [4] [5] resulting in stretching and ultimately breakage of the prelaminar nerve fibers since one end is attached to the soma of the RGC and the other end anchored in the pores of the LC. Only the prelaminar nerve fibers can be destroyed in an orderly tandem fashion since they are still loose and have not yet fastened in bundles in the pores of the LC. Once the nerve fibers are anchored in the pores of the LC, they can't be separated individually and thus their orderly tandem severance is not possible. Therefore, the LC may not be the site of injury in glaucoma as commonly believed.

\section{Why Are the Nerve Fibers Being Destroyed in an Orderly Fashion?}

The sinking of the LC and severance of the nerve fibers can explain their orderly destruction in glaucoma. As the LC sinks, the peripheral nerve fibers closest to the scleral edge are stretched and broken first, Figure 3. As a result, the next central fiber will move towards the periphery to occupy the space vacated by the preceding severed fiber and thus also get stretched and severed at the scleral edge.

In addition to the border tissue, the 360 degrees of nerve fibers also anchor the LC as roots anchor a tree. Thus, the severance of nerve fibers leads to further disc sinking. The cascade of severance of the nerve fibers and sinking disc would become self-propagated and will continue until all the nerve fibers have moved in an orderly tandem fashion to the scleral edge and get severed. This may explain the unstoppable nature of glaucoma despite maximum lowering of IOP. The severed segments undergo phagocytosis and thus will create empty spaces or excavation that we may be interpreting as cupping of the disc.

\section{Do We Have Evidence of Severance of Nerve Fibers?}

Progressive thinning of the RNFL in glaucoma can only be explained due to severance of nerve fibers as it is not occurring in non-glaucomatous optic atrophies. The arcuate retinal empty spaces [6] in glaucoma are due to severance and depletion of arcuate nerve fibers and notching due to their depletion at the site of their entry in the disc. All of the 360 degrees of nerve fibers are being severed simultaneously, however the arcuate fibers being fewer in number are depleted earlier, thus producing the arcuate field defects, Figure 4 and Figure 5. Notching at the poles of the disc is the initial excavation in the disc and a confirmatory sign of glaucoma. At this stage, the pathognomonic arcuate field defects will appear on perimetry.

The histology of the end-stage glaucomatous disc is not $100 \%$ cupped LC, but an empty crater left over after the phagocytosis of severed nerve fibers, Figure 6. Splinter hemorrhages and characteristic whitish pallor of the disc are due to severance of the vasculature which is also meeting the fate of nerve fibers. In contrast, the histology of the non-glaucomatous optic atrophy such as due to multiple sclerosis, reveals the presence of nerve fibers though atrophied and shrunken, Figure 7.

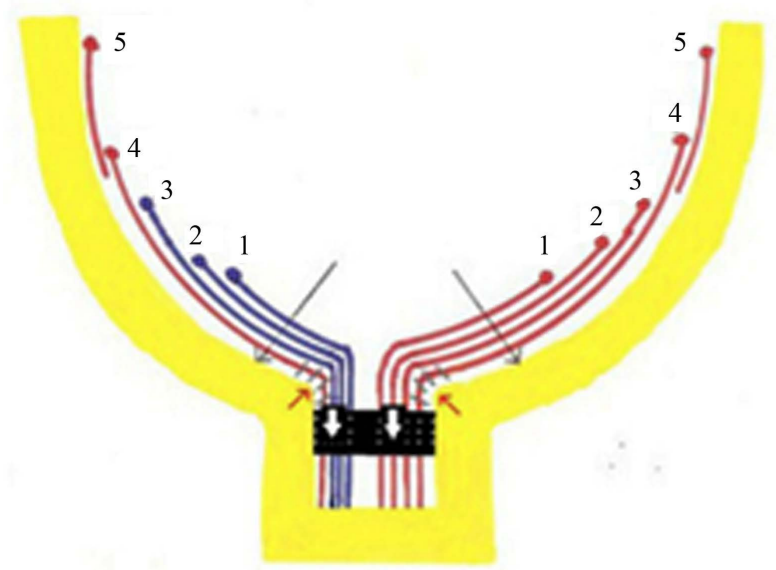

Figure 3. Note the sinking of the disc resulting in stretching and severing of the peripheral fibers. Most Peripheral fiber (5) has been severed and disappeared and this process will continue until central most fiber (1) has been severed. There will be movement of the central fibers to the periphery to occupy the space created by severance of the peripheral fibers. 


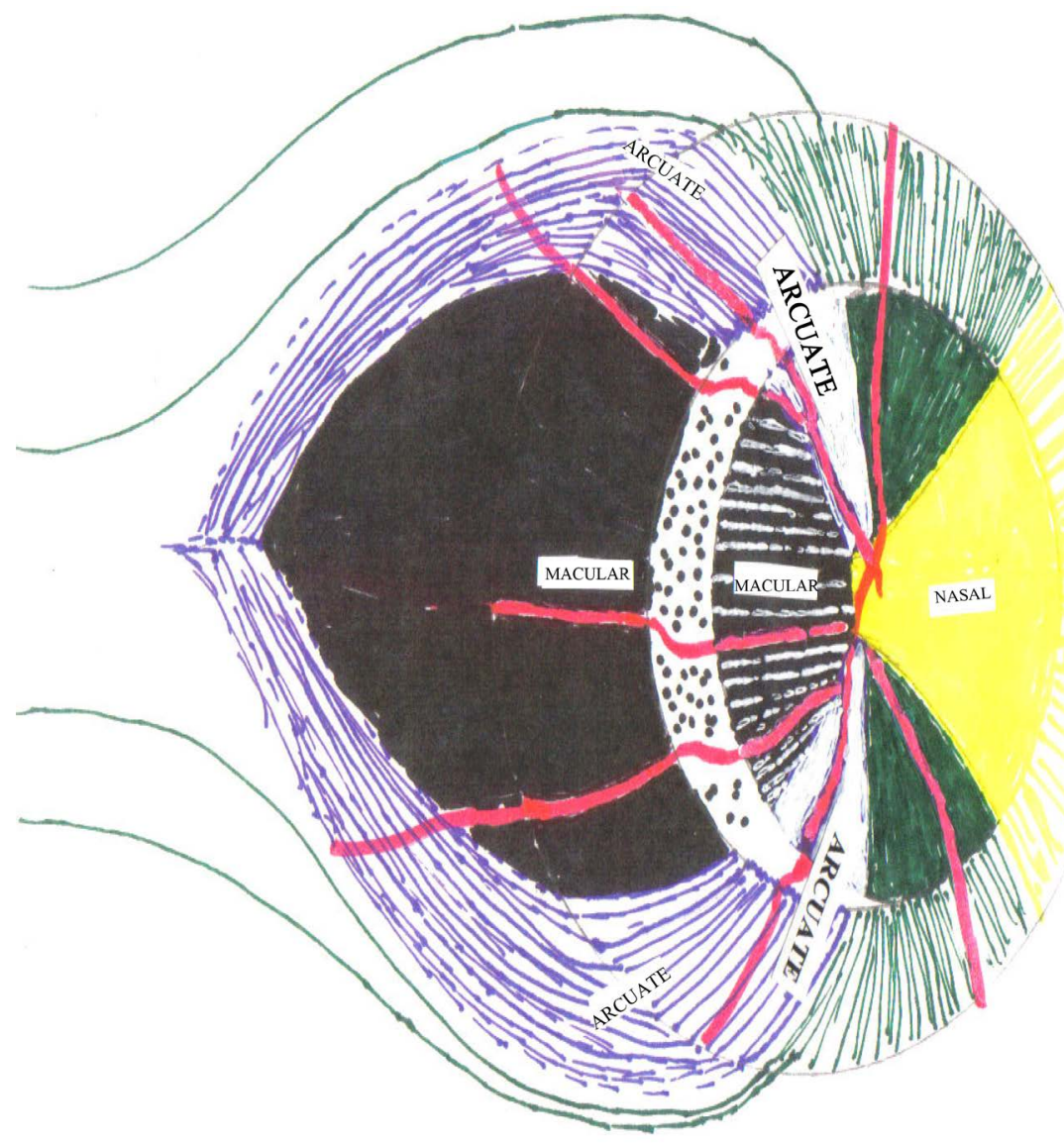

Figure 4. Due to temporal sinking all the temporal fibers (Macular, superior and inferior arcuate) are being axotomized. However, the arcuate fibers being fewer in number will be depleted earlier resulting in arcuate field defects as in Figure 5.

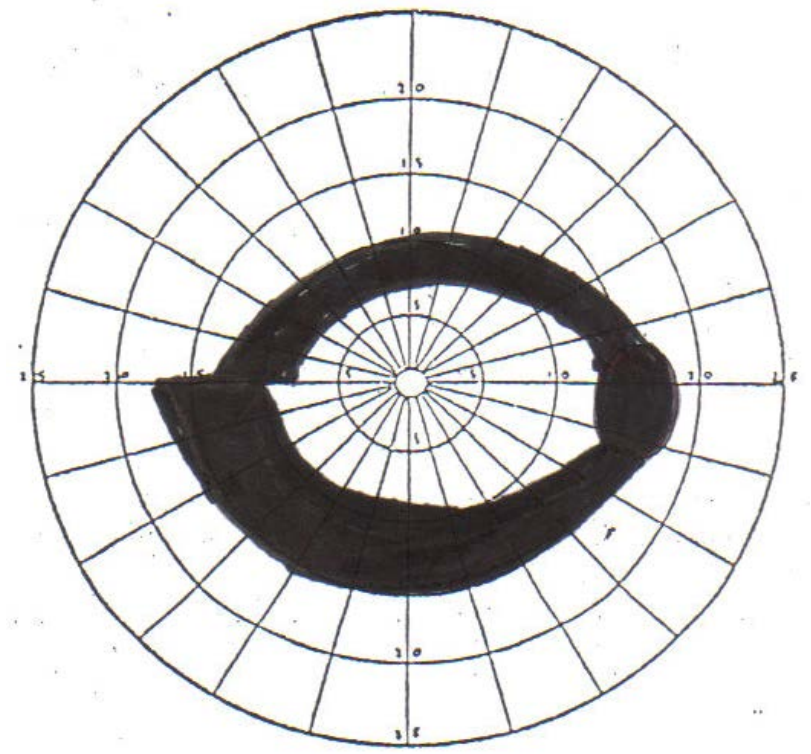

Figure 5. Right eye: Double arcuate/ring scotoma after arcuate fibers have been severed. Arcuate fibers being fewer in number compared to macular fibers, will be depleted earlier resulting in arcuate field defects. 


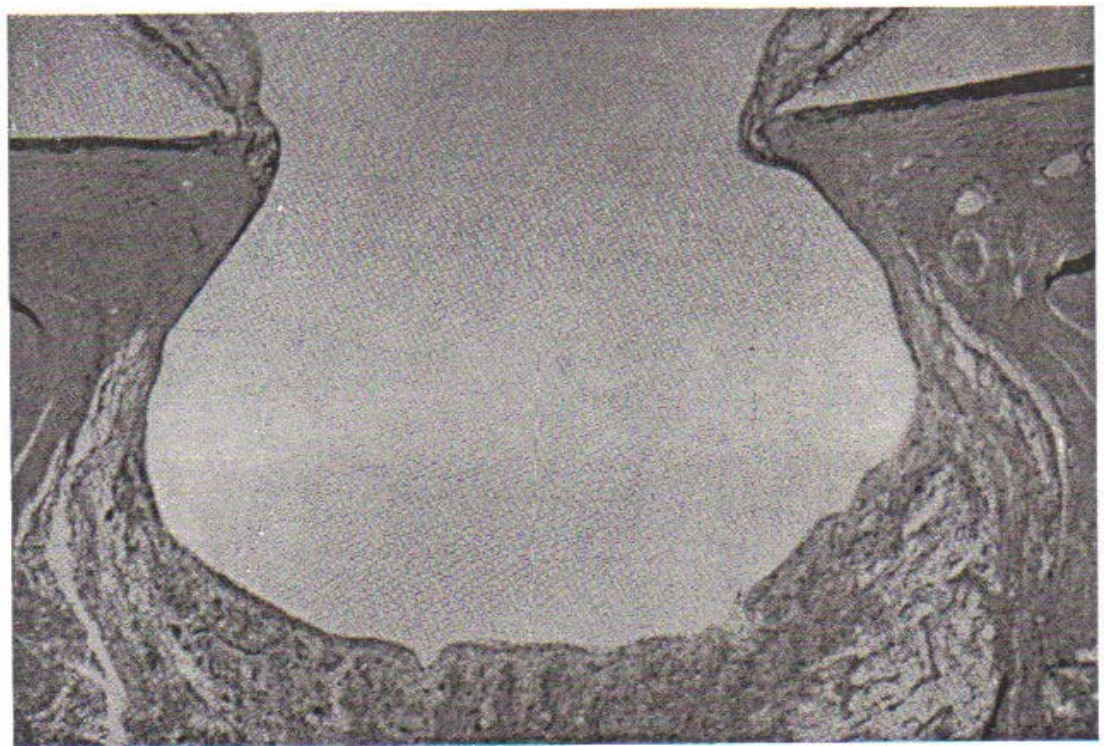

Figure 6. End-stage glaucomatous disc. Bean-pot excavation is not a deeply cupped disc/lamina but a left over crater which once housed the disc. The lamina is probably lying at the bottom of the crater after being emptied of all the nerve fibers after their severance. Kolker AE, Hetherington Jr J. Becker-Shaffer's diagnosis and therapy of the glaucoma, Mosby, 1976, p 146.

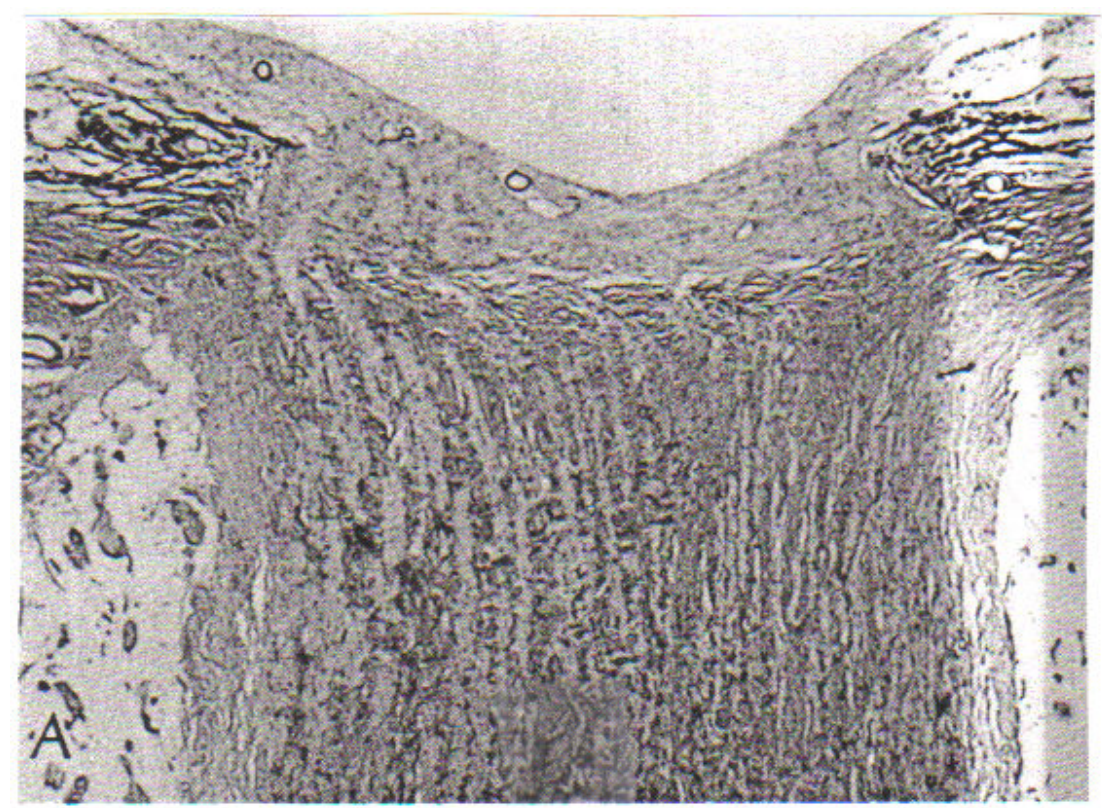

Figure 7. Flat disc atrophy due to multiple sclerosis. Note the nerve fibers though atrophied and shrunken are still present in contrast to glaucomatous disc. There is no excavation of the disc since there is no sinking and thus no severance of nerve fibers is occurring in flat disc atrophy. Copy from Yanoff, Ocular Pathology 1975.

\section{Conclusion}

The severance of the nerve fibers appears to be the missing piece in glaucoma mystery. The sinking disc and severance of nerve fibers is able to corroborate with their orderly destruction, a hallmark of glaucoma. In essence, the nerve fibers along with vasculature are being severed in glaucoma. Therefore, glaucoma may not be 
an optic disc neuropathy but an optic disc axotomy.

\section{References}

[1] Duke-Elder, S. and Barrie, J. (1969) Diseases of the Lens and Vitreous, Glaucoma and Hypotony. System of Ophthalmology, Vol. X1, Henry Kimpton, London, 385.

[2] Hasnain, S.S. (2006) Scleral Edge, Not Optic Disc or Retina Is the Primary Site of Injury in Chronic Glaucoma. Medical Hypothesis, 67, 1320-1325. http://dx.doi.org/10.1016/j.mehy.2006.05.030

[3] Hasnain, S.S. (2012) Can Glaucoma Be a Neurodegenerative Disease? Highlights of Ophthalmology. Panama, 40.

[4] Hasnain, S.S. (2010) Optic Disc May Be Sinking in Chronic Glaucoma. Ophthalmology Update, 8, 22-28.

[5] Yang, H. (2010) Optic Nerve Head (ONH) Lamina Cribrosa Insertion Migration and Pialization in Early Non-Human Primate Experimental Glaucoma. Poster Presentation ARVO Meeting, 3 May 2010.

[6] Hasnain, S.S. (2013) Arcuate Field Defects in Glaucoma Ophthalmology Update. 